\title{
Watt-level, all-fiber supercontinuum source based on telecom-grade fiber components
}

\author{
Jacek Swiderski $\cdot$ Maria Maciejewska
}

Received: 4 June 2012/Revised: 12 July 2012/Published online: 22 September 2012

(c) The Author(s) 2012. This article is published with open access at Springerlink.com

\begin{abstract}
Supercontinuum (SC) generation in a standard telecom fiber using $1 \mathrm{~ns}$ pulses of a 1,550-nm DFB laser amplified in a cascade of erbium and erbium/ytterbium fiber amplifiers is reported. The SC source operated at $200 \mathrm{kHz}$ repetition rate and delivered up to $2 \mathrm{~W}$ of average output power in the band of 1,300-2,500 nm with a diffraction limited beam. For the wavelengths over 1,650 nm, the output power of $1.1 \mathrm{~W}$ was recorded. The spectrum was very flat with the flatness of $<5 \mathrm{~dB}$ in the wavelength interval of 1.6-2.18 $\mu \mathrm{m}$. To the best of our knowledge, it is the first report on W-level SC generation obtained only in a standard single-mode fiber (SMF-28) with almost the entire spectrum in the eye-safe spectral region $(\lambda>1.4 \mu \mathrm{m})$ permitted by silicate glass transparency.
\end{abstract}

\section{Introduction}

A considerable interest in SC laser sources has been observed over the past two decades. This interest has manifested itself in a large number of books and papers related to this topic. In general, SC light can be obtained by pumping a piece of optical fiber with high intensity laser radiation. Different types of mode-locked lasers [1, 2], Q-switched lasers [3, 4] or CW lasers [5] are usually employed as pump sources and different nonlinear media, including silica-based fibers, appropriate for SC generation in the visible $[6,7]$, as well as fluoride [3, 8], tellurite [9], and chalcogenide [10] fibers suitable for mid-infrared operation have been used. It

J. Swiderski $(\bowtie) \cdot$ M. Maciejewska

Institute of Optoelectronics, Military University of Technology,

2 Kaliskiego Street, 00-908 Warsaw, Poland

e-mail: jswiderski@wat.edu.pl is worth noting here that majority of literature reports are related to SC in the visible and near-infrared range obtained with the use of silica photonic crystal fibers (PCFs) pumped by mode-locked lasers.

To develop a reliable, compact, low-cost, all-fiber SC generator that could satisfy market demands, all the SC components have to be fusion spliced and in that context fiber-based pump laser sources and silica-based fibers (as nonlinear media) are the best candidates for this purpose. Although spectral broadening in silica fibers is limited by strong material absorption in the mid-infrared region, they can be successfully used for SC generation in the wavelength range from the visible to $\sim 2.6 \mu \mathrm{m}$ wavelength [11] - the band that is interesting for LIDAR systems [12, 13], optical coherence tomography [14] or chemical sensing and microscopy [15].

Since mode-locked lasers are very expensive, complicated in construction, inflexible for output average power scaling up and require constant, periodic maintenance so as to ensure an optimal operation, a very interesting solution is to replace them with gain-switched semiconductor distributed feedback (DFB) lasers (generating ns or ps duration pulses) followed by a compact, commercially available (or easy to develop) cascade of erbium or erbium and erbium/ ytterbium amplifiers. In literature this approach is called Master Oscillator Fiber Power Amplifier configuration [16]. This concept is not a new one, and it was successfully utilized for SC generation and already reported in $[6-8,11,17]$. However, most of the reports were focused on SC generation in highly nonlinear (HiNL) fibers (characterized by higher nonlinear parameter $\gamma$ compared to conventional optical fibers) including fluoride fibers or dispersion shifted fibers. What must be noted is that significantly smaller attention has been given to $\mathrm{SC}$ generation in the mid-infrared range with the use of cheap and widely commercially available standard 
telecom single-mode fibers (SMF) pumped by nanosecond pulses.

In the present paper, we report on a compact, high brightness, ready-to-use SC source built with the use of a standard SMF (Corning, SMF-28) and a $1.5 \mu \mathrm{m}$ telecomgrade MOPA source seeded by a directly modulated DFB laser providing $1 \mathrm{~ns}$ pulses. The SC spectrum spreads mainly in the eye-safe spectral band from 1,300-2,500 nm and is characterized by a relatively flat spectral distribution of the intensity $(<5 \mathrm{~dB})$ in the wavelength interval of $\sim 1,600-2,200 \mathrm{~nm}$. The maximum achieved average power was $2 \mathrm{~W}$, measured for all spectral band and $1.1 \mathrm{~W}$ for wavelengths beyond $1.65 \mu \mathrm{m}$.

\section{SC source arrangement}

The SC source was configured as a MOPA system, as is shown in Fig. 1. The optical pulse train was generated with a semiconductor seed laser. The generated pulses were amplified in three amplifying stages formed by two corepumped erbium preamplifiers and a booster erbium/ytterbium-doped double-clad amplifier.

A pulsed DFB laser in a fiber-pigtail package with a built-in optical isolator protecting it from undesirable backcoming radiation was used as a seed. The seed operated at the wavelength of $1,550.12 \mathrm{~nm}$ and delivered a train of $1 \mathrm{~ns}$ pulses at a repetition rate of $200 \mathrm{kHz}$. The average output power for this duty cycle was $3 \mu \mathrm{W}$, which corresponds to the pulse energy and pulse peak power of $15 \mathrm{pJ}$ and $15 \mathrm{~mW}$, respectively. It is worth mentioning here that by applying the direct current modulation (phase modulation) of the seed it was possible to enlarge its emission linewidth by a chirp effect [18].

The preamplifiers utilized single-mode, single clad erbium-doped fibers with the core/clad diameter of $4 / 125 \mu \mathrm{m}$. The first one, of $1.5 \mathrm{~m}$ in length, was pumped by a laser diode delivering up to $500 \mathrm{~mW}$ of optical power at $976.4 \mathrm{~nm}$ wavelength, in co-propagating pump scheme, whereas the second one ( $1.3 \mathrm{~m}$ long) was pumped on both sides by two $976.3 \mathrm{~nm}$ laser diodes, each delivering up to
$500 \mathrm{~mW}$. The pump light was launched into the active Er-doped fibers with the use of 980/1,550 wavelength division multiplexers (WDMs).

The booster amplifier was built with a 2.4-m long single-mode double-clad erbium/ytterbium codoped fiber and provided a gain of $12.5 \mathrm{~dB}$. It had a $6.5 \mu \mathrm{m}$ diameter core with $0.19 \mathrm{NA}$, and the inner cladding diameter was $125 \mu \mathrm{m}$ with $0.45 \mathrm{NA}$. Its geometrical parameters were similar to those of a standard SMF-28 telecom fiber, thus simplifying fiber splicing process. Short pulses from the laser oscillator and the short length of the active fiber used in the booster amplifier prevented the onset of stimulated Brillouin scattering (SBS) - the major limiting factor in high peak-power single frequency fiber-based laser sources. The active fiber was pumped by one $10 \mathrm{~W}$ level (976 nm) pigtailed (105 $\mu \mathrm{m} / 0.22 \mathrm{NA}$ fiber core diameter/ NA) laser diode through a $(2 \times 1)+1$ multimode pump power combiner with a single-mode signal feedthrough at $1,550 \mathrm{~nm}$.

The seed and all amplifying stages were optically isolated by single-stage pigtailed optical isolators. Two $100 \mathrm{GHz}$ band-pass filters, placed between the amplifiers, were used to eliminate the out-of-band amplified spontaneous emission (ASE) generated by the Er-doped fiber amplifiers. A 99/1\% $2 \times 2$ tap coupler was spliced after the second preamplifier to monitor on-line the spectrum, pulse duration and power generated by the seed and the $\mathrm{Er}$ pre-amplifiers with $1 \%$ forward branch, whereas the backward $1 \%$ branch of the tap coupler was used to monitor the SBS signal emergence, coming from the booster amplifier. Since the booster amplifier was pumped in counter-propagation configuration and the $\mathrm{Er} / \mathrm{Yb}$ fiber provided total pump power absorption of $8.15 \mathrm{~dB}$, it was necessary to use a power cladding stripper (PCS) placed between the second Er preamplifier and the active $\mathrm{Er} / \mathrm{Yb}$ fiber, to remove $\sim 15 \%$ of unabsorbed pump power propagating in the clad towards the seed. The PCS provided $12 \mathrm{~dB}$ cladding attenuation for pump radiation and $0.3 \mathrm{~dB}$ signal insertion loss. The output signal from the MOPA system was directly launched into a piece of standard telecom fiber.
Fig. 1 Block diagram of an allfiber SC source

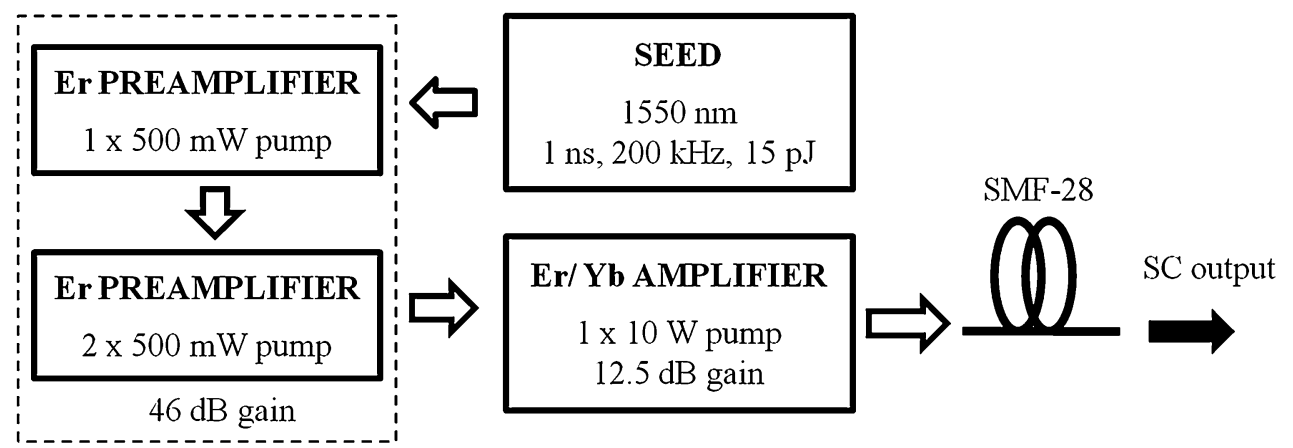


The design approach allowed the developed SC source to be cooled using forced air or conduction cooling. All the system components were spliced together, thus providing an all-fiber architecture for robustness, compactness and ease of manufacturing. This flexibility provides easy integration with a wide variety of systems used in different applications areas.

\section{Experimental results and discussion}

During the experiment, the seed and erbium amplifiers were operated at their maximum power, whereas the pump power launched into the booster amplifier was varied in the range from 0 to $10 \mathrm{~W}$. The system provided up to $58.5 \mathrm{~dB}$ total gain (the average output power of $2.14 \mathrm{~W}$ ). The fiber MOPA output was spliced with a standard SMF-28 having a length of $4.2 \mathrm{~m}$ (it was fusion spliced to the output fiber pigtail of the pump combiner). Since the two passive fiber pigtails of the pump power combiner have $20 \mathrm{~cm}$ in length, the total fiber SC length was $4.6 \mathrm{~m}$.

Figure 2 shows the SC spectrum recorded for several values of average output power. Spectral measurements were performed using Yokogawa AQ6375 optical spectrum analyzer (OSA) with measurement range of $1,200-2,400 \mathrm{~nm}$ and the grating spectrometer SP-2358 (Princeton Instruments) with a HCT detector (VIGO System, Poland) covering the spectral band of $2-5 \mu \mathrm{m}$. Then the two measured spectra were spliced together.

As can be seen in Fig. 2, higher pump power yields wider spectrum that can be achieved and for maximum pump power the spectrum extends from 1,300 to 2,500 nm, measured at $-70 \mathrm{~dB}$ level. It can also be noted that the spectrum is relatively flat in the wavelength interval of $\sim 1,600-2,200 \mathrm{~nm}$ with intensity variation smaller than

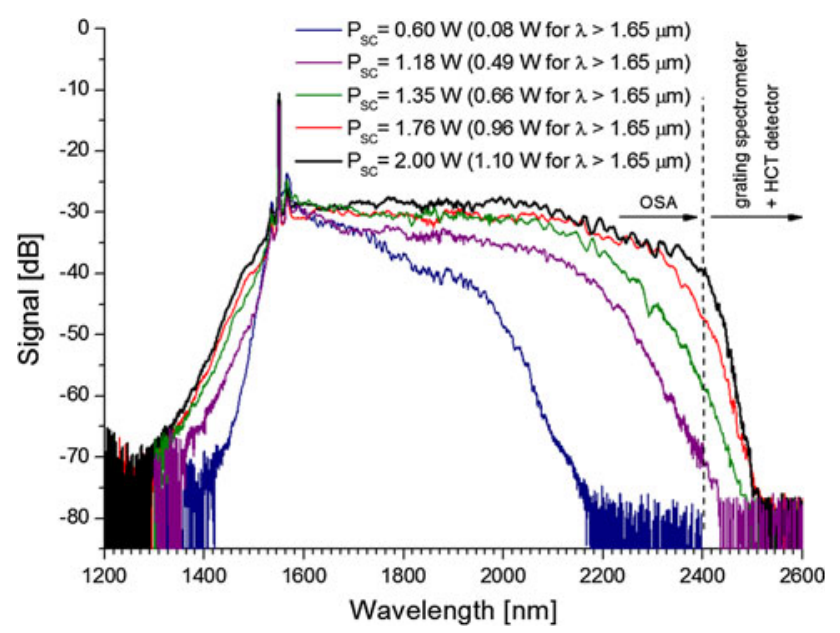

Fig. 2 Output spectrum of the SC source for different average output power
$5 \mathrm{~dB}$. The spectrum was also very stable in time in the whole wavelength range with deviation of $<3 \%$ which is very important for many applications. One can see that the intensity falls rapidly (for the case of maximum output power) for wavelengths higher than $2.4 \mu \mathrm{m}$, which is caused by the intrinsic losses of silicate glasses.

The average power of the SC spectra was measured by a power meter (Ophir, Laserstar) with thermal sensor response range of $0.19-20 \mu \mathrm{m}$, covering the entire SC spectra band. The achieved maximum average output power, including the peaks at $1,550 \mathrm{~nm}$, was measured to be equal to $2 \mathrm{~W}$ (Fig. 3). Since we did not use an autocorrelator for the measurement of output pulses temporal profile, we related the measured SC output power to the pump power launched into the booster $\mathrm{Er} / \mathrm{Yb}$ amplifier and not to pulse peak power. The power in the spectral range above $\lambda>1,650 \mathrm{~nm}$ (after a long-pass filter) was $1.1 \mathrm{~W}$ constituting $55 \%$ of the total output power. The deviation from linearity of the curve of the average output SC power versus power pumping the booster amplifier of the MOPA system occurs for pump power higher than $5 \mathrm{~W}$ (output SC power of $1.2 \mathrm{~W}$ ) for which the spectrum tail is shifted beyond $\lambda=2.4 \mu \mathrm{m}$. The drop of SC generation efficiency results from the fact that with the increase in pump power the SC power is distributed more and more towards longer wavelengths that experience larger material losses, and thus reducing the total output power.

Mechanisms responsible for SC generation in optical fibers have already been studied theoretically and experimentally and widely reported [e.g., 11, 19, 20]. Since a SMF is pumped in anomalous part of its dispersion characteristic, the main phenomena causing spectral broadening are modulation instability (MI) effect, leading to the creation of solitons with duration in the femtosecond range $[19,21]$, and soliton self-frequency shift caused by Raman

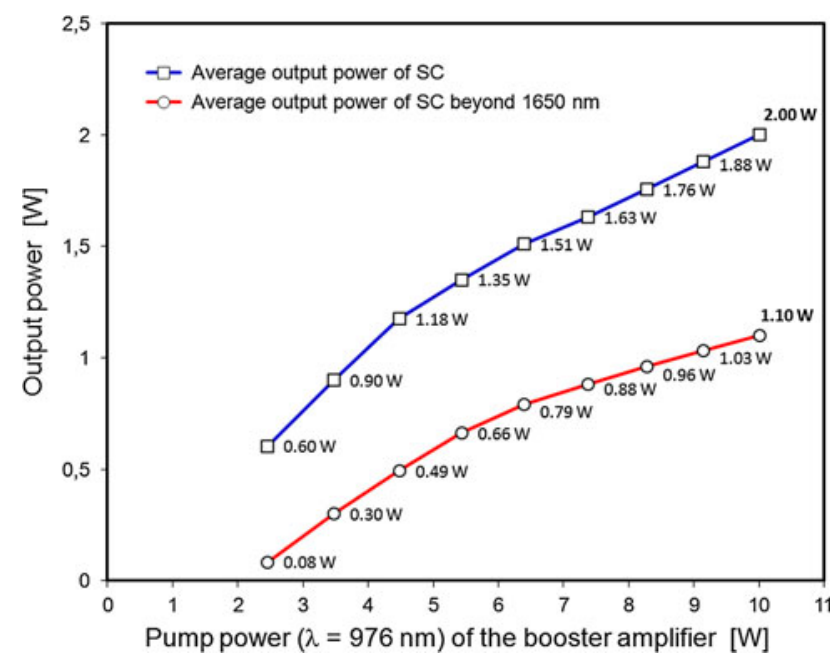

Fig. 3 Average SC power versus pump power of the booster amplifier 
scattering [11]. Indeed, the characteristic for MI process sidebands, separated by about $20 \mathrm{~nm}$ from the signal at $1,550 \mathrm{~nm}$, can be distinguished in Fig. 2. In the developed $\mathrm{SC}$ generator, the $1 \mathrm{~ns}$ pulses were broken into a train of multiple pulses with definitely shorter duration. In the experiment, we did not carry out any autocorrelation measurements, but the temporal profile of the output pulses was monitored using a fast photodetector with rising time of $<35 \mathrm{ps}$ (EOT, ET-5000F) and a sampling oscilloscope with $6 \mathrm{GHz}$ bandwidth (Tektronix, DSA70604). As can be seen in Fig. 4, the amplitude of output pulse is overmodulated with signatures of pulse splitting. Therefore, as emphasized in [6-8, 11], MI process can be effectively used for generation of sub-nanosecond pulses within $1 \mathrm{~ns}$ time gate and consequently, for obtaining broad SC spectrum in the similar way as in case of mode-locked lasers, however, contrary to mode-locked lasers, with high average output power at the same time.

The intensity profile of the output SC beam was measured with the use of a fully automated $M^{2}$ system using the beam propagation analyzer with a pyroelectric profiler (Ophir, NanoModeScan), making measurements according to the ISO 11146. Fig. 5 shows the beam quality factor measurement of the output SC beam centered at $\lambda=1,650 \mathrm{~nm}$ (FWHM band-12 nm). As can be seen, the output radiation is characterized by the near diffractionlimited beam with $M^{2}$ equal to 1.008 and 1.084 for $X$ and $Y$ axes, respectively.
Fig. 4 Temporal waveform of the output SC pulse

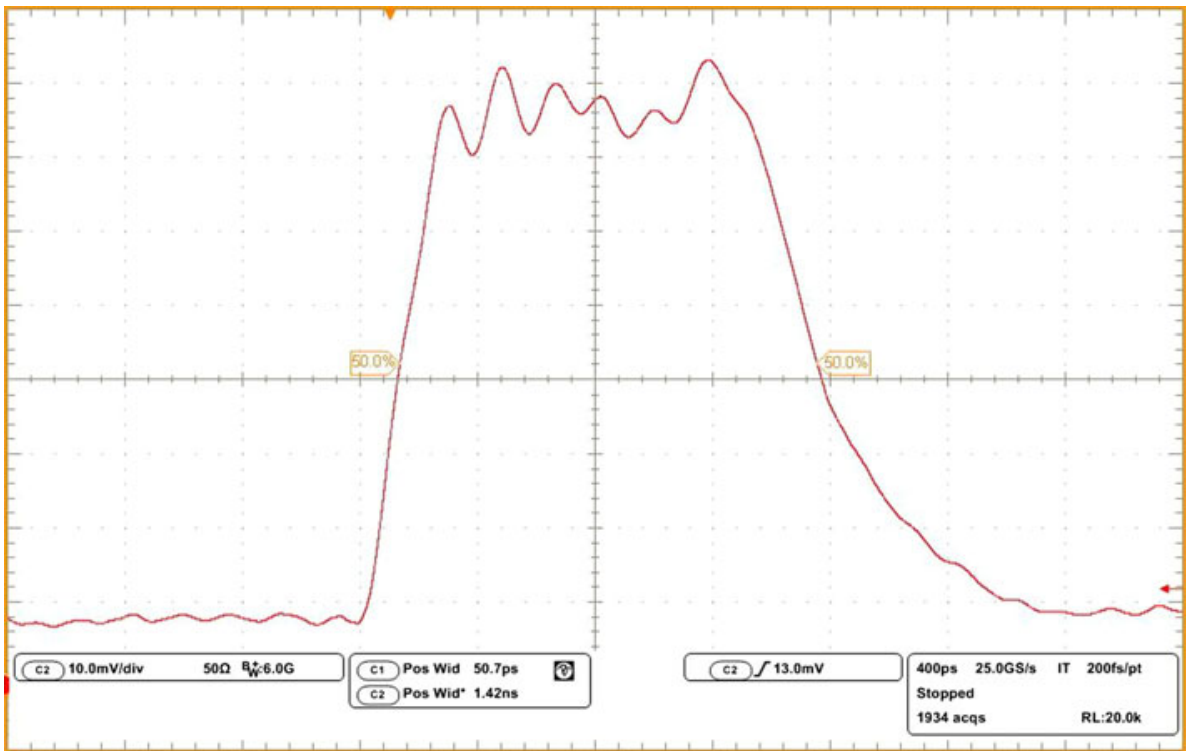

Fig. 5 Beam quality of the SC source: picture of output beam intensity profile at maximum power (a), $M^{2}$ measurement for $X$ axis (b), $M^{2}$ measurement for $Y$ axis (c)

(b)

(a)

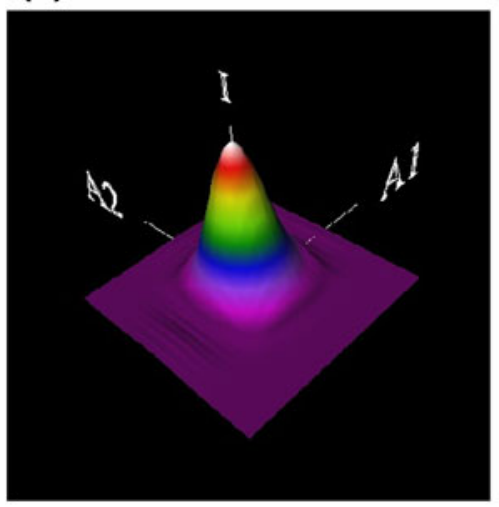

\section{(b)}

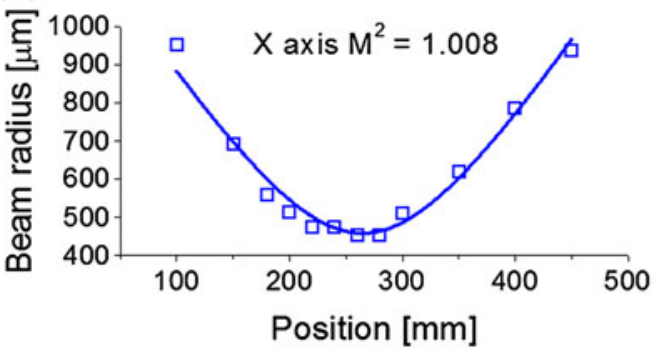

(c)

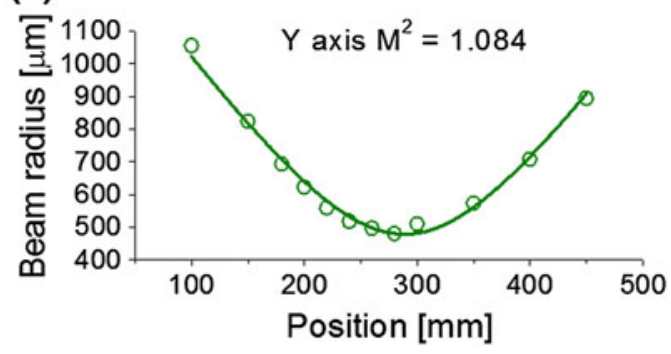




\section{Conclusions}

In summary, we demonstrated a compact, cost-effective, all-fiber, diffraction-limited, broadband SC source with the use of standard silica SMF pumped by $1 \mathrm{~ns}$ pulses $(\lambda=1,550 \mathrm{~nm})$ in the anomalous dispersion region. A spectrum spread from 1,300 to $2,500 \mathrm{~nm}$ was experimentally demonstrated. A maximum average SC power of $2 \mathrm{~W}$ was obtained and $55 \%$ of that power was contained in the band $\lambda>1.65 \mu \mathrm{m}$. The spectrum is characterized by $5 \mathrm{~dB}$ flatness in a wide wavelength range from $\sim 1.6$ to $\sim 2.2 \mu \mathrm{m}$. The output power can be further scaled up by applying more powerful pump MOPA source.

Acknowledgments This research has been supported by The Polish National Centre for Research and Development under project No. LIDER/04/198/L-1/09/NCBiR/2010.

Open Access This article is distributed under the terms of the Creative Commons Attribution License which permits any use, distribution, and reproduction in any medium, provided the original author(s) and the source are credited.

\section{References}

1. H. Hundertmark, S. Rammler, T. Wilken, R. Holzwarth, T.W. Hansch, P.S. Russell, Opt Express 17, 1919 (2009)

2. S.P. Stark, J.C. Travers, P. St, J. Russell, Opt Lett 37, 770 (2012)

3. M. Eckerle, C. Kieleck, J. Swiderski, S.D. Jackson, G. Maze, M. Eichhorn, Opt Lett 37, 512 (2012)

4. V.A. Kamynin, A.S. Kurov, V.M. Mashinsky, Laser Phys Lett 9 , 219 (2012)

5. A. Kudlinski, G. Bouwmans, O. Vanvincq, Y. Quiquempois, A. Le Rouge, L. Bigot, G. Melin, A. Mussot, Opt Lett 34, 3631 (2009)
6. S. Moon, D.Y. Kim, Opt Express 14, 270 (2006)

7. M. Kumar, C. Xia, X. Ma, V.V. Alexander, M.N. Islam, F.L. Terry, C.C. Aleksoff, A. Klooster, D. Davidson, Opt Express 16, 6194 (2008)

8. O.P. Kulkarni, V.V. Alexander, M. Kumar, M.J. Freeman, M.N. Islam, F.L. Terry Jr, M. Neelakandan, A. Chan, J Opt Soc Am B 28, 2486 (2011)

9. P. Domachuk, N.A. Wolchover, M. Cronin-Golomb, A. Wang, A.K. George, C.M.B. Cordeiro, J.C. Knight, F.G. Omenetto, Opt Express 16, 7161 (2008)

10. J. Hu, C. R. Menyuk, L. B. Shaw, J. S. Sanghera, I. D. Aggarwal, in Technical Digest Conference on Lasers and Electro-Optics, paper CThN6 (OSA 2009)

11. C. Xia, M. Kumar, M.-Y. Cheng, O.P. Kulkarni, M.N. Islam, A. Galvanauskas, F.L. Terry Jr, M.J. Freeman, D.A. Nolan, W.A. Wood, IEEE J Sel Top Quantum Electron 13, 789 (2007)

12. S. Kaasalainen, T. Lindroos, J. Hyyppa, IEEE Geosci Remote Sens Lett 4, 211 (2007)

13. Y. Chen, E. Raikkonen, S. Kaasalainen, J. Suomalainen, T. Hakala, J. Hyyppa, R. Chen, Sensors 10, 7057 (2010)

14. S. Ishida, N. Nishizawa, T. Ohta, K. Itoh, in Technical Digist Conference on Lasers and Electro-Optics, paper CThT4 (OSA 2010)

15. C.F. Kaminski, R.S. Watt, A.D. Elder, J.H. Frank, J. Hult, Appl Phys B 92, 367 (2008)

16. J. Swiderski, A. Zajac, M. Skorczakowski, Opto-Electron Rev 15, 98 (2007)

17. R. Calvani, R. Caponi, C. Naddeo, D. Roccato, Electron Lett 31, 1685 (1995)

18. D. Cotter, Elec Lett 18, 638 (1982)

19. G.P. Agrawal, Nonlinear Fiber Optics, 4 th edn. (Academic Press, New York, 2006)

20. J. M. Dudley, J. R. Taylor, Supercontinuum Generation in Optical Fibers (Cambridge University Press, 2010)

21. S.K. Turitsyn, A.M. Rubenchik, M.P. Fedoruk, Opt Lett 35, 2684 (2010) 